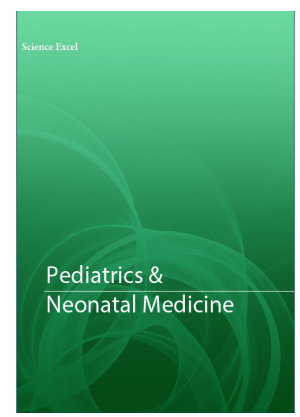

Correspondence

Yassegoungbe Monsoia Gildas

Pediatric Surgeon, Clinical Pediatric Surgery of the Centre National Hospital and Hubert Koutoukou Maga University of Cotonou Benin

E-mail: gildas.yassegoungbe@gmail.com

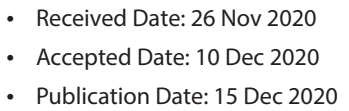

Copyright

(C) 2021 Science Excel. This is an openaccess article distributed under the terms of the Creative Commons Attribution 4.0 International license.

\title{
Lumbar Contusion: A Rare Cause of Voluminous Pseudocyst Pancreas in Children
}

\author{
Monsoia Gildas Yassegoungbe ${ }^{1 *}$, Codjo Serge Metchihoungbe ${ }^{1}$, Baudelaire \\ Romulus Assan', Amoussou Sedjro Clotaire Romeo Houegban', Aldrico \\ Pautin Covi', Mahunakpon Vihotogbe Léon Samuel Boris Gogan', Djifid Morel \\ Seto ${ }^{2}$, Houenoukpo Koco', Geraud Garcia Philemon Satingo Segbedji', Michel \\ Armand Fiogbe ${ }^{1}$
}

${ }^{1}$ Clinical Pediatric Surgery of the Centre National Hospital and Hubert Koutoukou Maga University, Cotonou, Benin ${ }^{2}$ Surgical Department, Zone Hospital of Allada, Allada, Benin

\begin{abstract}
The pseudo-cyst of the pancreas (PCP) is a rare affection in Africa, especially in children. We report the case of a 13 years old girl who has suffers a neglected lumbar trauma for 46 days and complain of abdominal pain and bloating. The positive examination is an asymmetrical abdomen, increased in volume as a whole. At palpation we note a voluminous mass slightly painful, mobile in relation to the superficial plan and adherent to the deep plan taking all the left hemi-abdomen overflowing the middle line measuring $12 \mathrm{~cm}$ of large axis and $10 \mathrm{~cm}$ of small axis, soft, renitent, dull to percussion. Abdominal CT allows to reveal a hypodense structure in contact with the body and tail of the pancreas to their anterior face to which it connects as a spur, repressing the digestive handles at the bottom and the stomach at the top. A laparotomy, internal bypass by a kysto-gastric anastomosis with a simple surgical follow-up, is performed.
\end{abstract}

\section{Introduction}

The fake pancreas cyst, currently called pseudo-cyst of the pancreas (PCP) is considered as a predominant entity in the pathology of the pancreas. It is a liquidian collection rich in pure or mixed pancreatic juice, contained in a neo-formed lodge without a clean wall made of disparate elements borrowed around the neighbouring organs [1]. It is most often extra pancreatic or in rare cases intra pancreatics. It is a rare condition in Africa, especially in children [2]. Etiologies identified are often acute lithiasic or alcoholic pancreatitis and an acute episode or acutization of chronic pancreatitis in adults or abdominal trauma, apanage of children as the case we present. The diagnosis of this pathology which was difficult in the past, is now easy thanks to the advances medical imaging. The particularity of our case is the seat of the contusion which is lumbar and the compressive character of the mass. External or internal drainage remains the principle of the cure of this pathology.

\section{Observation}

This is a 13 years old girl transferred from a nearby hospital for presenting a sub-occlusive syndrome. The onset of symptomatology was 5 days prior to admission marked by abdominal pain with fever and vomiting. This prompted a consultation in a health center where she was treated for febrile gastroenteritis for 2 days. The evolution was marked by persistent pain and fever, a cessation of materials and a progressive increase in the volume of the abdomen despite an unproductive evacuator enema. In this history, 46 days before the onset of symptoms, there was a post-accidental road trauma with a blunt reveal (iron bar) in the right lumbar region. The aftermath had been marked by right lumbar pain on the 3rd day posttraumatic and a febricula. These symptoms improved spontaneously without treatment on the 5th post-traumatic day; during these course, the child had not visited the hospital until the new symptoms appeared.

The positive admission examination showed an average general state (asthenia, weight loss), with constants within the limits of normal. The abdomen is asymmetrical, increased in volume overall predominating on the left. On palpation, a large, slightly painful mass was noted, rated at 4/10 on EVA, mobile in relation to the superficial plane and adherent to the deep plane, taking up the entire left hemi-abdomen beyond the midline. The mass was soft, rigid, with a dullness appearance on percussion and measured $12 \mathrm{~cm}$ long axis and $10 \mathrm{~cm}$ short axis. There was no auscultatory 


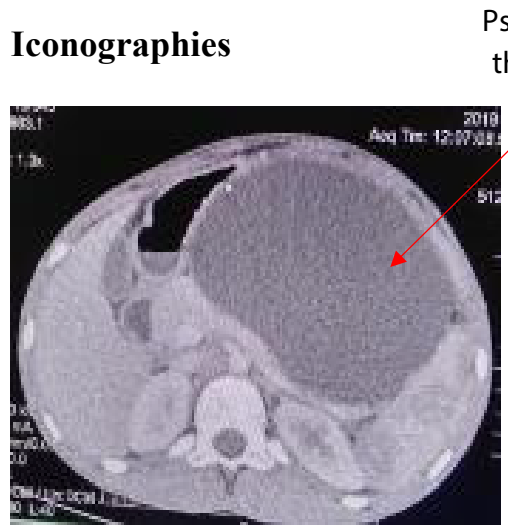

Pseudo cyst of the pancreas

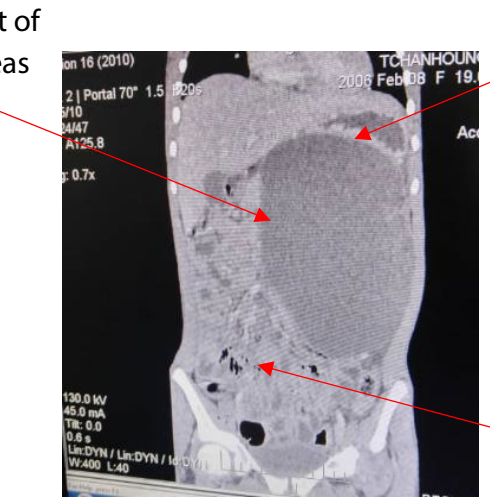

Stomach

repressed up
Hail handles and settler pushed down

Figure 1. Abdomino-pelvic scanner revealing a hypodense structure with a regular wall made heterogeneous by the presence of thin partition and measures $15.3 \mathrm{~cm}-9.3 \mathrm{~cm}, 17.2 \mathrm{~cm}$.

murmur or hydro-aerial noise. The umbilical perimeter was $66 \mathrm{~cm}$. The rectal examination was normal. On the right dorso-lumbar region, there was a small oval scar in the lumbar region, indicating lumbar impact. The rest of the examination was unremarkable.

Amylasemia and lipasemia were normal. On the abdominalpelvic CT scan, a hypodense structure with regular wall measured at $3 \mathrm{~mm}$ not raised after injection of contrast agent was noted, in contact with the body and the tail of the pancreas at their anterior face to which it connects in a spur. This structure was made heterogeneous by the presence of a thin wall and measured $15.3 \mathrm{~cm} * 9.3 \mathrm{~cm} * 17.2 \mathrm{~cm}$. The mass pushed the digestive coves downwards and the stomach upwards. There was no deep adenopathy or intraperitoneal effusion (Figure 1).

The diagnosis of post-traumatic pancreatic pseudocyst was retained and a surgical cure by laparotomy was indicated.

After hydroelectrolytic rebalancing, we induced a median incision under general anesthesia, overflowing into the sub umbilical area. At the coeliotomy a renal mass corresponding to a pseudocyst with cysto-parietal adhesions is reveal. Intraoperatively, an accidental rupture of the upper cystic wall in a zone close to the gastric wall is noted during the release of the cystoparietal adhesions. The breach is temporarily closed with Kelly forceps to facilitate dissection of the cyst wall. It is then widened at the end of dissection (Figure 2). The contents of the cyst are aspirated. It was a lemon-yellow liquid with a foamy appearance in the suction jar (saponification) indicating the pancreatic contents (Figure 3). After evacuation of the contents of the pseudocyst, we observe a retraction of its pouch and perform cystogastric anastomosis (Figures 4 and 5).

The immediate postoperative follow-up is simple with regression of transit disorders, a soft abdomen without palpable mass and good healing of the operative wound. The late consequences were marked by good weight gain, a calm, not unsightly scar. After a 30 -month follow-up with no recurrence, we can say that we attained a complete recovery of the patient.

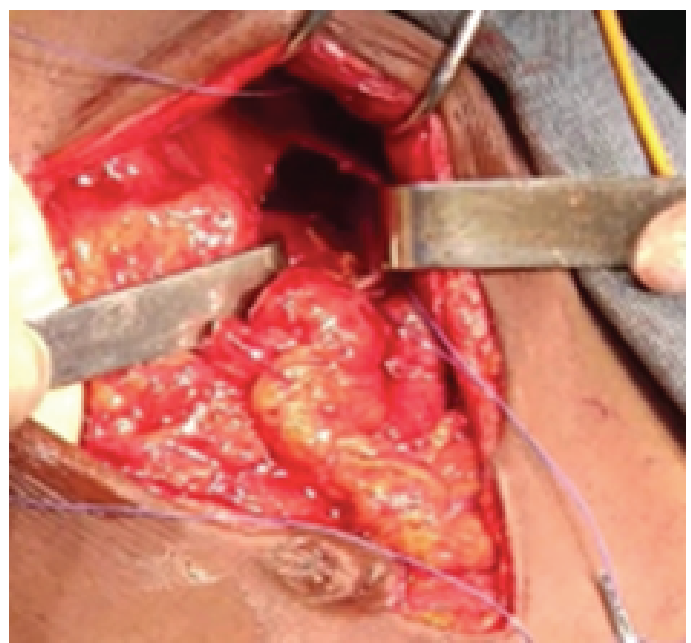

Figure 2. Breach closed first by Kelly's pliers and then widened at the end of the pocket dissection.

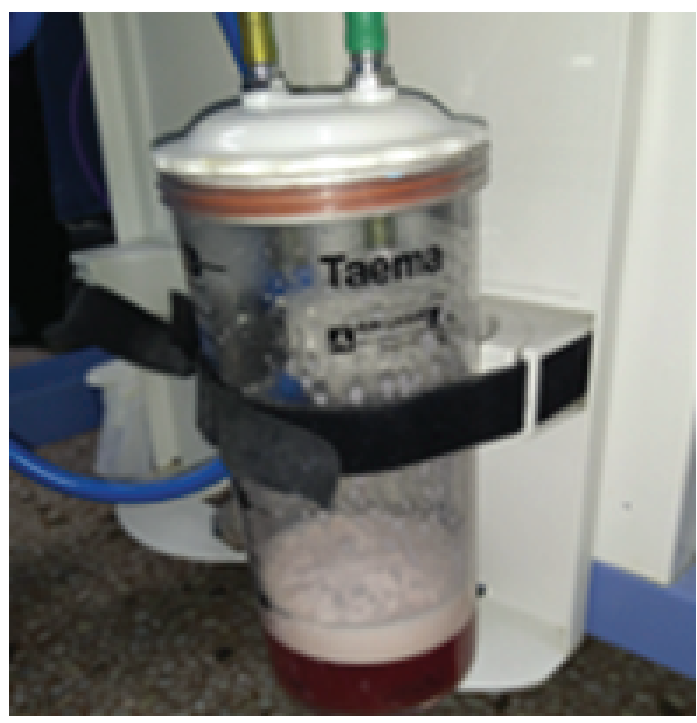

Figure 3. Emptying the cystic contents with a sparkling appearance in the suction jar. 


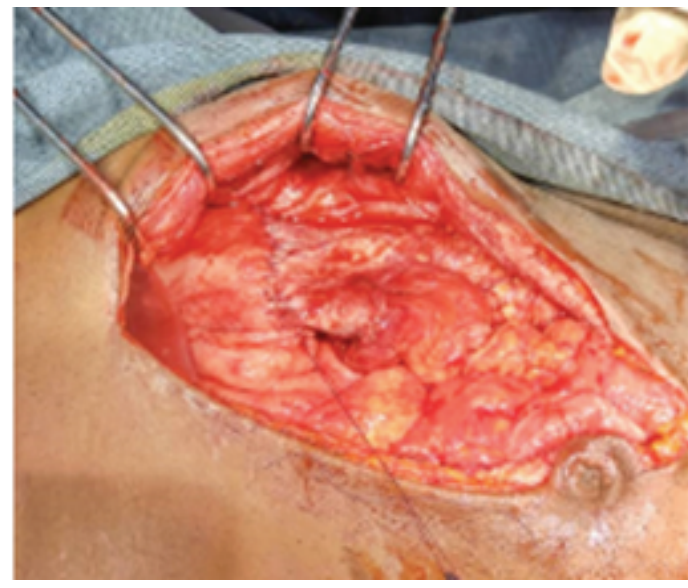

Table 4. Start of kystogastric anastomosis.

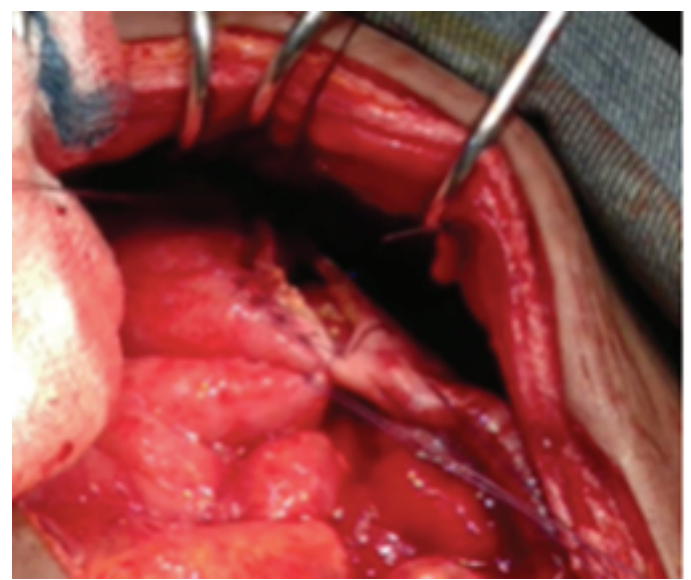

Table 5. End of kystogastric anastomosis.

\section{Discussion}

Pseudocysts of the pancreas (PCP) is a rare disease in children [3]. While pseudocysts in adults are among the complications of chronic pancreatitis in $20-80 \%$ of cases, depending on the series, mostly of ethylic origin, closed abdominal trauma remains the most frequent etiology of PCP in children [4-5]. Acute pancreatitis ranks second [5]. This makes it possible to distinguish in pediatric surgery between two pathogenic forms of pseudocyst: traumatic and non-traumatic. In our case, it is a lumbar contusion.

This condition can be seen at any age. The average age of most published pediatric series is between 6 and 7.5 years $[2,3]$. In our clinical case, the child is a female above the predominant age. Though statistics shows that the female sex is often less affected than the male sex. [6].

PCPs seem to result from a rupture of a pancreatic duct (most often the secondary ducts), following trauma or a parenchymal contusion allowing pancreatic juice to flow into the abdomen. This collection is rapidly circumscribed by the neighboring organs and then a fibrous tissue is formed which serves as the envelope of the cyst [7]. It can also be a complication of post-traumatic pancreatitis. The latter manifests itself by the occurrence of constant abdominal pain after a latent interval that can range from 4 days to 4 weeks. In our case, the interval was 46 days.

In the case of a post-traumatic etiology, falling off a bicycle with the handlebars touching the epigastrium was the most common event $[1,4,5]$. In the case we report, the abdominal impact was right dorsal-lumbar which is unusual. The occurrence of the pseudocyst after this impact can hardly be explained by the retroperitoneal situation of the pancreas. The delay in the onset of the first abdominal pain and its spontaneous resolving character call for pancreatic contusions rather than a manifestation of a hypothetical post-traumatic pancreatitis. From a clinical point of view, the difficulty is to link unusual manifestations to the pancreas or those revealed by a complication [8], which may explain the diagnostic wandering in the health center from which it was referred to us. Thus, the mode of revelation is diverse, dominated by abdominal pain and the presence of an abdominal mass [4]. This mass is often epigastric or in the left hypochondrium (50\% of cases in the literature). Its hardness, in case of a pseudocyst under tension, can mimic a solid tumor [4]. The volume of this mass occupying practically the left hemi-abdomen in our patient, as well as its compressive character of the handles pushed downwards, explains the sub-occlusive syndrome (abdominal pain, vomiting, stoppage of material) which constituted the mode of revelation of this disease. Sometimes this mass can regress spontaneously or evolve and become symptomatic [4]. In biology, hyperamylasemia or hyperlipasemia does not plead in favor of a pseudocyst whatever the cause may be, only prolonged and post-pancreatitis hyperamylasemia can plead in favor. This is not the case in this child where amylasemia and lipasemia were normal.

Abdominal ultrasound is the most cost-effective morphological examination for PCP. It offers a specificity of $97.5 \%$ and a sensitivity of $87.9 \%$ [2]. The abdominal CT scan is a more efficient examination. It gives much sharper images and can be requested if there is any doubt about the diagnosis. These two complementary exams have shown their usefulness and complementarity in making the diagnosis of PKP for monitoring purposes; they have helped us to have a precise imaging of the lesion. We did not perform an opacification of the pancreatic ducts to check the communication of the cyst with the excretory ducts of the pancreas.

The spontaneous evolution of PCP, apart from organ compression, can lead to complications such as rupture (intra digestive, intra peritoneal or intra vascular), intra-cystic bleeding by erosion of a vessel or infections. The treatment is both medical, with antiemetics, analgesics and solutes, and surgical. The presence of symptoms or complications is the first indication for surgical management [7]. The rupture in our case was not a complication because it had been revealed intraoperatively during dissection, which proves that the wall of this pseudocyst was already weakened and pre-perforative. This could explain the sharp pain.

Concerning the management, it is a question of external or 
internal derivations by laparotomy or endoscopy. Percutaneous external shunts or marsupialization are indicated in cases of symptomatic PCP that is highly infected, thin-walled, friable and not easily sutured wall [9]. Although rarely indicated, cases of cyst regression under this treatment have been reported. The factors for good progression were mainly the rarity of Wirsung's canal lesions in children, but this treatment is long and perilous and provides infection [10], which led us to choose an internal shunt in our case.

Internal drainage by endoscopy or laparotomy is determined by the location of the cyst. However, regardless of its location, it is always possible to drain PCP surgically from a hollow viscera (stomach, duodenum or jejunum) [11]. The drainage cavity will depend on the intraoperative findings. In view of the accidental rupture of the cyst near the stomach in this child, we decided to proceed with a cystogastric bypass. It should be noted that, according to the series, the shunt is often mostly cysto-jejunal, but the gastric shunt also has a good prognosis [3]. The simple post-operative sequelae confirm the effectiveness of this technique despite the volume of the mass and the intraoperative rupture requiring gastric bypass.

\section{Conclusion}

Pseudocyst of the pancreas after lumbar contusion in children is rare. Any trauma to the abdomen must be monitored to quickly reveal these lesions and avoid complications. Despite the accidental rupture, internal cysto-digestive internal shunt remains the adequate treatment.

\section{References}

1. Light L. Surgical pathology. 3rd edition Masson, Paris, 1978. 673-677.

2. Fall B, Thognon $\mathrm{PH}$, Ndiaye $\mathrm{M}$, et al. Le pseudokyst du pancréas : diagnostic, traitement. About seven observations. Dakar medical 1990;35:177-181.

3. Bap AP, Ngom G, Sankale AA, Fayel L. Management of pseudocyst of the pancreas in children at the CHU Aristide le Dantec DAKAR: about 7 cases. Médecine d'Afrique noire 2006, 53(11):630-632.

4. Arvieux C, Letoublon C. Pancreatic trauma. Elsevier SAS 2005;10:1016.

5. Lesur G, Levy P, Sauvaget A, Vilgrain V, Belghiti J, Bernardes P. Pseudocysts of the pancreas: natural history and therapeutic indications. Gastroenterol Clin Biol 1994;18:880-8.

6. Cherrabi H, Aboueljaoud H, Diallo Harouna A, El Madi A, Khattala K, Bouabdallah Y. Pseudocyst of the pancreas in children: about 7 cases : Pan African Medical Journal. 2019;32:77.

7. Habashi S, Draganov PV. Pancreatic pseudocyst. World Journal Gastroenterol 2009 January 7;15(1):38-47

8. Faik M, Halhal A, Oudanane M, Housni K, Elbaroudi S, Tounsi A. False cysts of the pancreas about 13 cases. Médecine du Maghreb 2000 (79).

9. Kagan JR . Reyes HM. Asokan S. Pseudocvst of the pancreas in childhood, current advance in diagnosis. Arch Surg 1981;116:1200-1203.

10. Giakoustidis A, Antoniadis N, Giorgakis I, Tsoulfas G, Dimitriadis E, Giakoustidis D. Pancreatic pseudocyst in a child due to blunt andominal trauma during a soccer Game. HIPPOKRATIA 2012;16(1):71-73. 